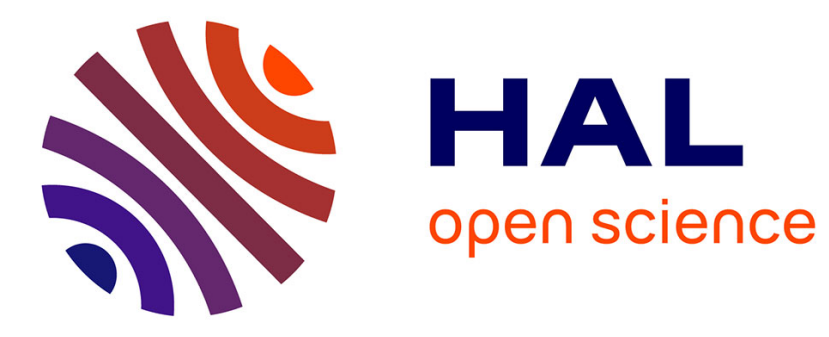

\title{
Data inversion for hyperspectral objects in astronomy
}

Thomas Rodet, François Orieux, Jean-François Giovannelli, Alain Abergel

\section{To cite this version:}

Thomas Rodet, François Orieux, Jean-François Giovannelli, Alain Abergel. Data inversion for hyperspectral objects in astronomy. First IEEE GRSS Workshop on Hyperspectral Image and Signal Processing, Aug 2009, Grenoble, France. 10.1109/WHISPERS.2009.5289008 . hal-00416573

\section{HAL Id: hal-00416573 \\ https://hal.science/hal-00416573}

Submitted on 5 Mar 2020

HAL is a multi-disciplinary open access archive for the deposit and dissemination of scientific research documents, whether they are published or not. The documents may come from teaching and research institutions in France or abroad, or from public or private research centers.
L'archive ouverte pluridisciplinaire HAL, est destinée au dépôt et à la diffusion de documents scientifiques de niveau recherche, publiés ou non, émanant des établissements d'enseignement et de recherche français ou étrangers, des laboratoires publics ou privés. 


\section{DATA INVERSION FOR HYPERSPECTRAL OBJECTS IN ASTRONOMY}

\author{
T. Rodet, F. Orieux, J.-F. Giovannelli
}

Laboratoire des Signaux et Systèmes

Supélec, 91192 Gif-sur-Yvette, France.

orieux, rodet, giova@lss.supelec.fr

\section{ABSTRACT}

We present an original method for reconstruction of hyperspectral objects (two spatial and one spectral dimensions) from data provided by the infrared slit spectrograph on board the Spitzer Space Telescope. The originality of the work lies in the fact that both measurement model and inversion method are tackled in continuous (spatial and spectral) variables. The method is built in a deterministic regularization framework and enable to achieve both deconvolution and over-resolution. Results show that the method is able to evidence spatial structures not detectable by means of conventional methods. The spatial resolution is shown to be improved by a factor 1.5 . We discuss our data processing approach for the new generation of infrared to millimeter space observatories launched in 2009 (Herschel and Planck).

\section{INTRODUCTION}

The aim of the spectral imaging work presented here is to reconstruct over-resolved objects with two spatial (angular) dimensions $(\alpha, \beta)$ and one spectral dimension $\lambda$. The work is illustrated thanks to the data provided by the Infrared Spectrograph (IRS) [1] on board the american Spitzer Space Telescope launched in 2003.

Data were acquired by means of a slit spectrograph, described in detail in part 2. Telescope pointing selects a direction of space $\alpha$ by means of the slit. The photon flux is then dispersed by a diffraction grating. Information about the spatial dimension $\alpha$ and the spectral dimension $\lambda$ is thus acquired. The second spatial dimension $\beta$ results form sky scanning (modification of telescope pointing).

The optical behaviour of the system is a function of the wavelength, so, natural data resolution is wavelength dependent (see Eq. (1)). Moreover, a phenomenon of spectral aliasing also appears for the shortest wavelengths.

The problem to be solved is thus one of inverting spectral aliasing (i.e., over-resolution [2]) based on a finite number of discrete data provided by a complex system. The proposed solution relies on a precise instrument model (optics

\footnotetext{
${ }^{0}$ J.-F. Giovannelli is in the Laboratoire de l'Intégration du Matériau au Système (IMS) Université de Bordeaux 1 since september 2008
}

\author{
A. Abergel \\ Institut d'Astrophysique Spatiale \\ Université Paris Sud-11, 91405 Orsay, France \\ abergeleias.u-psud.fr.
}

and sensing) based on integral equations w.r.t. the continuous variables $(\alpha, \beta$ and $\lambda)$. The model input is naturally a continuous variable object $\phi(\alpha, \beta, \lambda)$ and the output is a finite set $\boldsymbol{y}$ of discrete data items. The inverse problem, i.e., object reconstructing with three continuous variables from the discrete data comes within the regularization framework. It is founded on penalization by uses of a semi-parametric format based on object decomposed into a family of functions.

The major contribution of the paper relies on a continuous variable framework:

1. a model for the entire measurement system w.r.t. continuous variables enables a faithful description of the physical phenomena involved in the acquisition.

2. a continuous variable inversion method based on penalization in order to favour smooth solutions.

Computing the model output requires six integrals (two for the response of the optics, two for the grating response, and two for the sensor integration) and the decomposition of the three dimensional object over a family of Gaussian functions allows five of these six integrals to be explicitly stated.

\section{CONTINUOUS DIRECT MODEL}

The aim of the instrument model is to reproduce the data, $\boldsymbol{y}$, acquired by the imager for a given incoherent flux $\phi(\alpha, \beta, \lambda)$. It includes: (1) primary mirror (aperture), (2) truncation due to a rectangular slit, (3) dispersion by a grating and (4) distortion and sensor integration. The total model provides the discrete data $\boldsymbol{y}$ details is given in [3].

Aperture diffraction - Under standard hypotheses, the propagation of a light wave through an aperture is described by FRESNEL diffraction: it is modeled as a convolution of the input flux $\phi$ and the well-known Airy Point Spread Function (PSF) noted $h_{a}$. In the focal plane, the flux $\phi_{f}$, is then written in integral form

$$
\phi_{f}\left(\alpha^{\prime}, \beta^{\prime}, \lambda\right)=\iint \phi(\alpha, \beta, \lambda) h_{a}\left(\alpha-\alpha^{\prime}, \beta-\beta^{\prime}, \lambda\right) \mathrm{d} \alpha \mathrm{d} \beta
$$


Slit and diffraction grating - Ideally, the grating gives a diffracted wave with an output angle $\theta$ linearly dependent on the wavelength $\lambda$. In a more accurate model, the dependence becomes more complex. The response of the grating centred on mode $m$ ( $m=0,1, \ldots)$ can, with some approximations, be written as a squared cardinal sine centred on $m / a$

$$
h_{r}\left(\theta, \beta^{\prime}, \lambda\right)=B \operatorname{sinc}^{2}(\pi L((\theta-\lambda-m / a))
$$

where $L$ is the width of the grating and $a$ the grid step (distance between grooves). Since the flux is incoherent, the output of the grating writes as an integral over $\beta^{\prime}$ and $\lambda$

$$
\phi_{r}\left(\alpha^{\prime}, \theta\right)=\iint_{\left|\beta^{\prime}\right| \leq \gamma / 2} \phi_{f}\left(\alpha^{\prime}, \beta^{\prime}, \lambda\right) h_{r}\left(\beta^{\prime}, \lambda, \theta\right) \mathrm{d} \beta^{\prime} \mathrm{d} \lambda
$$

where $\gamma$ is the angular slit width (5.6 arcseconds).

Sensor integration - After dispersion through the grating according to the wavelengths, the flux is focused on the sensor. It is composed of square detectors and modelled by integration of the flux $\phi_{r}$ on square areas of side $d$

$$
y(i, j)=\int_{i d}^{(i+1) d} \int_{j d+e_{i j}^{1}}^{(j+1) d+e_{i j}^{2}} \phi_{r}\left(\alpha^{\prime}, \theta\right) \mathrm{d} \alpha^{\prime} \mathrm{d} \theta .
$$

The integration limits are modified by the $e_{i j}^{n}$ in order account for flux distortions.

Complete model - Combining (1), (2) and (3) yields a continuous model:

$$
\begin{aligned}
& y(i, j, q)=\mathcal{A} \iiint \iiint \phi\left(\alpha-\Delta_{\alpha}(q), \beta-\Delta_{\beta}(q), \lambda\right) \\
& h_{a}\left(\alpha-\alpha^{\prime}, \beta-\beta^{\prime}, \lambda\right) \operatorname{d} \alpha \mathrm{d} \beta h_{r}\left(\theta, \beta^{\prime}, \lambda\right) \mathrm{d} \beta^{\prime} \mathrm{d} \lambda \mathrm{d} \alpha^{\prime} \mathrm{d} \theta
\end{aligned}
$$

for the instrument, where $\left(\Delta_{\alpha}(q), \Delta_{\beta}(q)\right)$ is the pointed direction and $\mathcal{A}$ is a scale factor.

We then have developed a model linking the continuous object $\phi(\alpha, \beta, \lambda)$ and discrete data. It is linear but non shift invariant due to grating output dependence on the wavelength.

\section{DECOMPOSITION OVER A FAMILY AND GAUSSIAN APPROXIMATION}

Now we have a direct model that describes the data acquisition from sky. To get a good representation of the information restored by the method, the sky model needs to be adapted. On the opposite, a good choice of the sky model in combination of the direct model equation can reduce the amount of computation necessary to simulate a data acquisition. These constraints led us to consider band limited functions class for $\phi$. This class of functions can be represented without loose of information with the base of sinus cardinal functions. In this case the sampling period and the width of the sinus cardinal are fixed by the frequency limit.
The fact that the method aim to restore a sky with improved resolution led us to fix the frequency limit greater than the cut off frequency of the sensor. The choice of the frequency limit is still an open question. It's depend on the sampling frequency of the sensor, the acquisition protocol or the potential of the method. We fix this value to approximately twice the natural sampling frequency of the sensor since the shift between two acquisitions are the half of the slit width.

If we chose the sinus cardinal decomposition, the computation of the direct model becomes very prohibitive. Consequently, in conjunction of the approximation of the direct model equation, we choose Gaussian functions in spatial dimension that best approximate the corresponding sinc functions. This allow explicit resolution of the integral in the direct model.

Decomposition over a family of Gaussian functions - The flux $\phi$ is a continuous function decomposed over a family of separable functions:

$$
\begin{aligned}
\phi(\alpha, \beta, \lambda)=\sum_{k, l, m} x(k, l, p) \\
\Pi\left(\alpha-k T_{\alpha}\right) \Phi\left(\beta-l T_{\beta}\right) \Gamma\left(\lambda-p T_{\lambda}\right)
\end{aligned}
$$

where $x(k, l, p)$ are the decomposition coefficients, $T_{\alpha}, T_{\beta}$, $T_{\lambda}$ and $\sigma$ are determined by the frequency limit and

$$
\begin{aligned}
\Pi(\alpha) \Phi(\beta) & =\frac{1}{2 \pi \sigma^{2}} \exp \left(-\frac{1}{2} \frac{\alpha^{2}+\beta^{2}}{\sigma^{2}}\right) \\
\Gamma(\lambda) & =\delta(\lambda) .
\end{aligned}
$$

With such decomposition, the inverse problem becomes one of estimating a finite number of coefficients $x(k, l, p)$ from discrete data. If the $y(i, j, q)$ and $x(k, l, p)$ are gathered in vectors $\boldsymbol{y}$ and $\boldsymbol{x}$ respectively, the equation (4) can be formalized as a vector matrix product $\boldsymbol{y}=\boldsymbol{H} \boldsymbol{x}$.

Gaussian approximation of impulse responses - Equation (1) comes down to convolutions of a squared Bessel function and Gaussians. This integral is not explicit and, in order to carry out the calculations, the PSF is approximated by a Gaussian with a standard deviation $\sigma_{\lambda} \approx \lambda / 2$. The relative error $\operatorname{err}_{L k}=\|$ Bessel - Gaussian $\left\|_{k} /\right\|$ Bessel $\|_{k}$ is small $\left(e r r_{L 2}=0.15 \%, e r r_{L 1}=5 \%\right)$. We can conclude that most of the energy of the squared Bessel function is localized in the primary lobe. Finally, the result of the convolution of two Gaussian functions is a standard one and is also a Gaussian.

The presence of the slit means that integral (2) is bounded over $\beta^{\prime}$ and is not easily calculable. Since the preceding expressions use Gaussian functions, we approximate the squared cardinal sine by a Gaussian to make the calculations easier: $\sigma_{s}$ is determined numerically by minimizing the quadratic error between the Gaussian kernel and the squared cardinal sine, which gives for our instrument $\sigma_{s} \approx 25.5 \mathrm{~m}^{-1}$. The relative errors made are larger than 
the Bessel case $\left(e r r_{L 2}=0.43 \%, e r r_{L 1}=10.7 \%\right)$, but this Gaussian approximation of the grating response allows the flux $\phi_{r}$ coming out of the grating to be known explicitly.

\section{INVERSION}

The previous sections build the relationship between the object coefficients and the data. The problem of input (object) reconstruction is a typical ill-posed inverse problem and the literature on the subject is abundant. We want to reconstruct relatively smooth objects (clouds of galactic dust). To do this, we introduce an a priori smoothness through a penalty term (the last term of the Eq. (7)).

The proposed inversion method resorts to linear processing. It is based on conventional approaches described in books such [4]. These methods rely on a quadratic criterion

$$
J(\boldsymbol{x})=\|\boldsymbol{y}-\boldsymbol{H} \boldsymbol{x}\|^{2}+\mu_{\alpha \beta}\left\|\boldsymbol{D}_{\alpha \beta} \boldsymbol{x}\right\|^{2}+\mu_{\lambda}\left\|\boldsymbol{D}_{\lambda} \boldsymbol{x}\right\|^{2} .
$$

It involves a least squares term and two penalize terms $\boldsymbol{D}_{\alpha \beta}$ and $\boldsymbol{D}_{\lambda}$ concerning the differences between neighbouring coefficients: one for the two spatial dimensions and one for the spectral dimension. They are weighted by $\mu_{\alpha \beta}$ and $\mu_{\lambda}$, respectively. The estimate $\hat{\boldsymbol{x}}$ is chosen as the minimizer of this criterion. It is thus explicit and linear with respect to the data

$$
\hat{\boldsymbol{x}}=\left(\boldsymbol{H}^{\mathrm{t}} \boldsymbol{H}+\mu_{\alpha \beta} \boldsymbol{D}_{\alpha \beta}^{\mathrm{t}} \boldsymbol{D}_{\alpha \beta}+\mu_{\lambda} \boldsymbol{D}_{\lambda}^{\mathrm{t}} \boldsymbol{D}_{\lambda}\right)^{-1} \boldsymbol{H}^{\mathrm{t}} \boldsymbol{y}
$$

In practice, it cannot be calculated on standard computers, because the matrix to be inverted is too large. The solution $\hat{x}$ is therefore computed by a numerical optimization algorithm. Practically, the optimization relies on a standard gradient descent algorithm. More precisely, the direction descent is a approximate conjugate gradient direction [5] and the optimal step of descent is used.

\section{RESULTS}

Real data - Each data contain 23 acquisitions composed of $38 \times 128$ values. To obtain a over-resolved reconstruction, we describe our volume with 587264 gaussians distributed on a cartesian grid $74 \times 62 \times 128$. The spatial $(\alpha, \beta)$ sampling step is equal to a quarter slit width, and the spectral dimension is uniformly sampled between the wavelengths 7.4 and $15.3 \mu \mathrm{m}$. The reconstruction is computed after setting the regularization coefficients $\mu_{\alpha \beta}$ and $\mu_{\lambda}$ empirically. Too low a value for these coefficients produces an unstable method and a quasi explosive reconstruction. Too high a value produces images that are visibly too smooth. A compromise found by trial and error led us to $\mu_{\alpha \beta}=0.3$ and $\mu_{\lambda}=0.7$. The ratio between $\mu_{\alpha \beta}$ and $\mu_{\lambda}$ is also based on our simulation. However, we cannot compare the regularization coefficients between the simulated and the real case, since the size of the problem modifies the weight of the norm in the Eq. (7). Practically, we take large value for the regularization coefficients, and we gradually reduce the value up that we are seeing noise.
Our results (Fig. 1(b) ) can be compared with those obtained with (Fig. 1(a) from [6]). A comparison of Fig. 1(a) and 1(b) clearly shows that our approach provides more resolved images that bring out more structures than the conventional approach. Note, in particular, the separation of the two filaments on the left part of the Fig. 1(b) obtained with our method, which remains invisible after conventional processing. For comparison, Fig. 1(c) shows the same object observed with the Infrared Array Camera (IRAC) of the Spitzer Space Telescope which has a better native resolution since it observes at a shorter wavelength $(4.5 \mu \mathrm{m})$. Here the same structures are observed, providing a strong argument in favour of the reality of the results provided by our method.

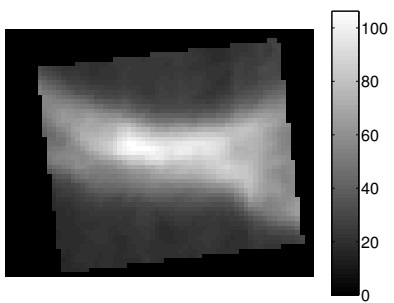

(a)

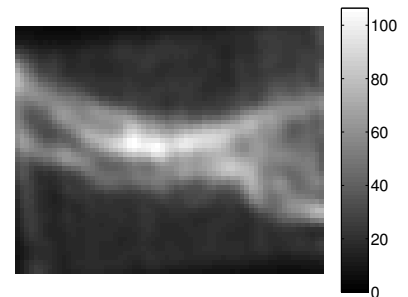

(b)

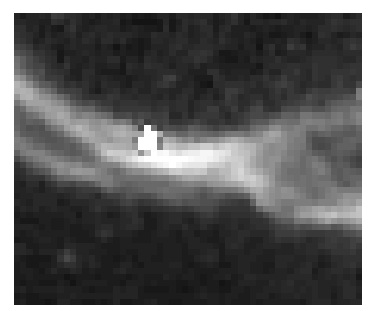

(c)

Fig. 1. Object reconstruction $\phi$ representing the Horsehead nebula: (a) image estimated at $11.37 \mu \mathrm{m}$ by the conventional method (b) image estimated at $11.37 \mu \mathrm{m}$ by our method, (c) image obtained with the Infrared Array Camera IRAC on board the same telescope having better resolution at $4.5 \mu \mathrm{m}$.

Study of resolving power of our approach - This part is devoted to numerical quantification of the gain in angular resolution provided with our method, using the Rayleigh criterion, which is frequently used by astrophysicists: for the smaller resolvable detail, the first minimum of the image of one point source coincides with the maximum of another. In practice, two point sources with the same intensity and a flat spectrum are considered to be separated if the minimal flux between the two peaks is lower than 0.9 times the flux at the peak positions. The resolution is studied in the $\beta$ direction only as this is the direction in which the subslit scan is performed.

Two point sources are injected, at positions $\beta_{1}$ and $\beta_{2}$, respectively (see Fig. 3 ). The corresponding data are simulated, and the reconstruction $\hat{\phi}(\beta)$ is performed. As explained above, the two point sources are considered to be separated if 
$\hat{\phi}\left(\left[\beta_{1}+\beta_{2}\right] / 2\right) \leq 0.9 \times \hat{\phi}\left(\beta_{1}\right)$. The resolution is defined as the difference $\delta=\beta_{2}-\beta_{1}$ at which the two point sources start to be separated.

Point sources are simulated for a set of differences $\delta$ between 2.4 and 5.4 arcseconds and simulations are performed in the configuration of the real data (signal to noise ratio, energy). A number of reconstructions has been obtained. The ratio between the values of the reconstructed function at $\beta_{1}$ and $\left(\beta_{1}+\beta_{2}\right) / 2$ is calculated as a function of the difference $\delta$ between the two peaks. Results are shown in Fig. 2 .

The computed resolution is 3.4 arcseconds (see Fig. 2(a)) and 5 arcseconds (see Fig. 2(b)) for our method and the conventional method, respectively.

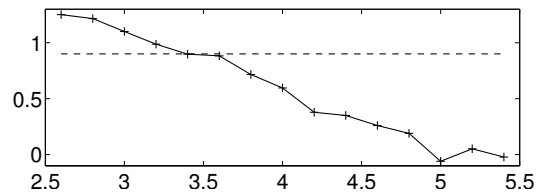

(a)

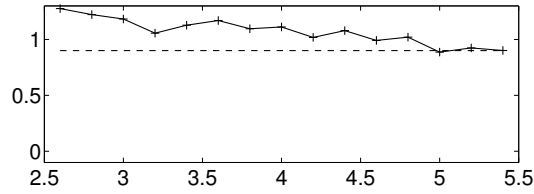

(b)

Fig. 2. Resolution of our method: the curve represents the ratio of the intensity at one peak to the intensity between the two peaks as a function of the distance between the peaks in arcseconds. The resolution is read at crossing of this curve and the dotted line (the ratio is 0.9). (a) Results obtained with our method. (b) Results obtained with the conventional method

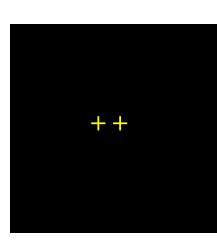

(a)

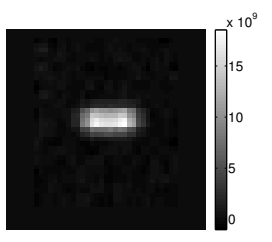

(b)

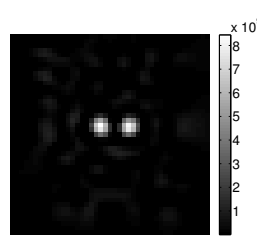

(c)
Fig. 3. Two peaks reconstruction for different $\delta$ : (a) Visualisation of the peaks position for $\delta=5.4$ arcsecond resp., (b) reconstruction with the conventional method and (c) reconstruction with our over-resolution method.

\section{CONCLUSIONS AND FUTURE WORKS}

We have developed an original method for reconstructing the over-resolved 3D sky from data provided by the IRS instrument. Firstly this method is based on a continuous variable model of the instrument based on a precise integral physical description. Secondly, a decomposition of the continuous variable object over a family Gaussian functions, results in a linear relationship. Thirdly, an inversion is done in the framework of deterministic regularization based on a quadratic criterion minimized by a gradient algorithm.

First results on real data show that we are able to evidence spatial structures not detectable using conventional methods. The spatial resolution is improved by a factor 1.5. This factor should increase using data taken with a motion between two successive acquisitions smaller than half the slit width.

Our approach leads to a linear model between the decomposition coefficients and the data. Our goal is to apply it for the next generation of infrared to millimeter space observatories (HERSCHEL, JWST, PLANCK, ...), since the limitation in angular resolution will be a critical issue. For the long wavelength imager of Herschel (SPIRE), we are currently developing a full model of the instrument, including the optics, the bolometer sensors, and the continuous motion of the telescope for large scale mapping. This model will be used to derive inverted maps. We are also working on the Mid Infra Red Instrument (MIRI) for the James Webb Space Telescope (JWST). We estimate high resolution Point Spread Functions with an unprecedented accuracy using "microscanning" measurements (sub-pixel scanning of a point-source on the focal plane) taken during ground-based optical tests Amiaux et al. 2008 "Development approach and first infrared test results of JWST/Mid Infra Red Imager Optical Bench", SPIE, 2008

This is the first step to obtain over-resolution images from the data which will be sent from the sky by the JWST.

\section{REFERENCES}

[1] J. R. Houck et al., "The infrared spectrograph (IRS) on the Spitzer space telescope," ApJS, vol. 154, pp. 18-24, September 2004 .

[2] S. C. Park, M. K. Park, and M. G. Kang, "Super-resolution image reconstruction: a technical overview," IEEE Trans. Signal Processing Mag., pp. 21-36, May 2003.

[3] Thomas Rodet, François Orieux, Jean-François Giovannelli, and Alain Abergel, "Data inversion for over-resolved spectral imaging in astronomy," IEEE J. of Selec. Topics in Signal Proc., vol. 2, no. 5, pp. 802-811, October 2008.

[4] Jérôme Idier, Ed., Bayesian Approach to Inverse Problems, ISTE Ltd and John Wiley \& Sons Inc., London, 2008.

[5] E. Polak, Computational methods in optimization, Academic Press, New York, NY, 1971.

[6] M. Compiègne, A. Abergel, L. Verstraete, W. T. Reach, E. Habart, J. D. Smith, F. Boulanger, and C. Joblin, "Aromatic emission from the ionised mane of the Horsehead nebula," Astronomy \& Astrophysics, vol. 471, pp. 205-212, 2007. 